\title{
Embedding linearly ordered sets in real lexicographic products
}

by

\author{
I. Fleischer (Waltham, Mass.)
}

In a previous paper, the anthor has studied linearly ordered sets isomorphic to subsets of the real numbers; here representation by lexicographic products is treated. Sets embeddable in countable products are characterized as containing no uncountable well-ordered or inversely well-ordered sequence. Subsets of finite products are treated in greater detail; the uniqueness of their representation is also investigated.

For a mapping $\varphi$ of a linearly ordered set $L$ into a linearly ordered set $L^{\prime}$, we shall use the term monotone to mean that $\varphi$ is monotone nondecreasing; if $\varphi$ is strictly monotone or monotone increasing, we shall say it effects an embedding of $L$ in $L^{\prime}$; or if also $L^{\prime}$ is completely covered by $\varphi$, that it is an isomorphism of $L$ with $L^{\prime}$. The inverse image of a single element under a monotone mapping is an interval; that is, a set which contains with any two elements also all elements between. Conversely, if $L$ is partitioned into disjoint intervals, the set $L^{\prime}$ of these intervals inherits from $L$ a unique linear order, while the mapping $\varphi$ which sends every element on the interval containing it, is monotone.

Let $L$ and $L^{\prime}$ be linearly ordered sets. Their lexicographic product is, by definition, their Cartesian product ordered by appeal to the first differing component. Explicitly, $L \times L^{\prime}$ consists of pairs $\left(x, x^{\prime}\right)$ with $x \in L$, $x^{\prime} \in L^{\prime}$ under the ordering convention: $\left(x, x^{\prime}\right)<\left(y, y^{\prime}\right)$ means $x<y$ or $x=y$ and $x^{\prime}<y^{\prime}$. Clearly the definition can be extended to any finite number of factors presented in a definite order; and indeed to infinitely many factors provided that for any two elements in the product "first differing component" has an unambiguous meaning; in other words, provided that the set indexing the components is well-ordered.

We recall that a cut in a linearly ordered set $L$ is a decomposition of $L$ into two disjoint non-empty intervals. Equivalently, a cut is a monotone mapping of $L$ on the two element set. Every linearly ordered set of more than one element has cuts; the intervals of a cut, insofar as they do not consist of a single element, may be cut again, effecting a monotone mapping of $L$ into the lexicographic product of the two element set with 
itself. This process of cutting an interval may be continued, indeed, evem transfinitely often inasmuch as the intersection of intervals is an interval and will only terminate when all intervals consist of a single element, at which stage the associated monotone function is an embedding. Thus, every linearly ordered set may be embedded in a product of two element. sets. (This result is due to Sierpiński [1].)

Now, if uncountably many cuts were used to effect the embedding. then there exists an uncountable nested strictly decreasing sequence: of intervals. For we may choose from the first cut one of the intervals. which is cut uncountably often thereafter, and then proceed by transfinite induction in this way past any countable ordinal. It is then a simple. matter to construct by transfinite induction an uncountable well-ordered or inversely well-ordered sequence of elements of $L$. Thus, if $L$ contains. no such sequence, it was necessarily embedded in a countable product.

It will be recalled that the first uncountable ordinal $\Omega$ is characterized as an uncountable well-ordered set, every proper initial interval of which is countable. It follows that every terminal interval is isomorphic to $\Omega$.

Suppose that $\varphi$ is a monotone real-valued function of $\Omega$. Because the monotone image of a well-ordered set is well-ordered, and in view of the denseness of the rationals, $\varphi$ can take on only countably many distinct values; since countable subsets of $\Omega$ are bounded, $\varphi$ is ultimately (i. e., for some terminal interval) constant. More generally, let $\varphi$ map $Q$ monotonically into a countable real lexicographic product. Since the projection of a product on its first component is monotone, one can find a terminal interval of $\Omega$ on which the first component of $\varphi$ is constant. Each terminal interval being isomorphic to $\Omega$, there will be a still smaller terminal interval on which the first two components of $\varphi$ are constant, and so on. Countably many terminal intervals of $\Omega$ always have a nonroid intersection; since only countable limit ordinals occur in the indexing set of the product, one concludes by transfinite induction that $\varphi$ is ultimately constant. In particular, in a countable lexicographic product of copies of the real numbers, every well-ordered subset is countable. In the same way (or by noting that such a lexicographic product is antiisomorphic with itself), one establishes that every inversely well-ordered subset is countable.

THEOREM 1. A necessary and sufficient condition that a linearly ordered set be embeddable in a countable real lexicographic product is that each of its well-ordered or inversely well-ordered subsets be countable.

We shall next address ourselves to a closer investigation of embeddability in finite real lexicographic products.

Let $L$ be a linearly ordered set. The binary relation $\varrho$ valid for exactly those pairs $x, y$ for which the interval lying between $x$ and $y$ is embeddable in the real numbers is readily seen to be an equivalence relation whose equivalence classes are intervals. Thus, $\varrho$ is induced by a monotone mapping of $L$ on a linearly ordered set $L^{\prime}$. If $L$ is the monotone image of a subset $S$ of real lexicographic $n$ space, then the equivalence induced by $\varrho$ in $S$ identifies at least all elements differing only in the last component (the monotone image of a real subset being real). It follows that $L^{\prime}$ is the monotone image of the set obtained from $S$ by identifying last components; that is, of the subset of lexicographic $(n-1)$ space made up of first $(n-1)$ components of elements of $S$. The equivalence $o$ may also be introduced in $L^{\prime}$ and defines a monotone mapping of $L^{\prime}$ on the linearly ordered set of its equivalence classes; and so on, but not ad infinitum. For it follows easily by ordinary induction that the procedure applied to a monotone image of a subset of lexicographic $n$ space will yield the one point set after at most $n$ iterations.

Conversely, the validity of this last condition ensures embeddability in lexicographic $n$ space in the presence of the condition of Theorem 1. For the absence of uncountable well-ordered or inversely well-ordered subsets in $L$ yields by transfinite induction that every subset contains a countable subset cofinal in both directions (i. e., generating the same interval). This being so in particular for the equivalence classes of $\varrho$, one deduces that these are each embeddable in the real numbers. Choice of an embedding for every class effectively furnishes an embedding of $L$ in the lexicographic product of $L^{\prime}$ with the real numbers. The resnlt now follows by ordinary induction.

THEOREM 2. A necessary, and for sets satisfying the condition of Theorem 1 also sufficient, condition for the embeddability of $L$ in real lexicographic $n$ spaee is that the equivalence $\varrho$ applied successively to the sets of its own equivalence classes, shall yield after at most $n$ iterations the universab equivatence.

The embedding achieved in the proof has the characteristic that the last component of the image is made as large as possible: in precise terms, the equivalence obtained by identifying last components in terms of any other embedding is finer than $o$. If this property is specified for the image of $L$ as well as for those of the successive sets of equivalence classes in the lower dimensional spaces, then the embedding is unique, except for the arbitrariness associated with the representation of each equivalence class by real numbers: that is, for fixed $x_{1}, \ldots, x_{i-1}, x_{i+1}, \ldots, x_{n}$ one has the freedom to replace the linearly ordered set of $x_{i}$ for which $\left(x_{i}, \ldots, x_{i}, \ldots, x_{n}\right) \in L$ by any real subset isomorphic to it.

Among equivalences on $L$ with real equivalence classes, $\varrho$ may be identified as that one for which every interval of $L^{\prime}$ contains uncountably many equivalence elasses with more than one element. The proof will be omitted. 
That equivalence classes of one element may occur even for the embedding of the theorem may be seen by referring to the lexicographic plane with the $F$-axis except for the origin removed.

The uniqueness considerations valid in finite products no longer hold in the infinite case. For example, let $L$ be a subset of the lexicographic product over the positive integers, $\theta$ a real number occuring as the first component of an element of $L$, and $I$ a real interval containing $\theta$. There exists an isomorphism of the elements less than $\theta$ into those less than $I$ as Trell as one of the elements greater than $\theta$ into those exceeding $I$; let such an isomorphism be applied to all first components other than $\theta$. For elements whose first component is $\theta$, let the second components be mapped into $I$ (by some isomorphism of the real numbers into $I$ ) and all succeeding components be mored back one index. The result is an isomorphism of $L$ with another subset of the product. If $L$ is the subset of all elements with irrational first component, then both it and its image appear in all finite dimensional products as required for the previous uniqueness conclusions, but thein first components are clearly not isomorphic.

\section{Reference}

[1] W. Sierpinski, Cardina and ordinal numbers, Warszawa 1958.

Reģu par la Rédaction le 18. 2. 1960

Sur les familles d'ensembles infinis de nombres naturels

par

\section{W. Sierpiński (Warszawa)}

F étant une famille donnée d'ensembles, le problème se pose de trouver les conditions pour qu'il existe au moins un ensemble ayant arec tout ensemble de la famille $F$ un et un seul élément commun, respectivement un nombre fini non nul d'éléments communs.

Dans le cas où la famille $F$ est formée d'ensembles non vides disjoints, l'existence d'un ensemble ayant un et un seul élément commun avec tout ensemble de cette famille résulte de l'axióme du choix. Or, la question se pose d'étudier le cas où les ensembles de la famille $F$ ont deux à deux ar plus un élément commun, respectivement un nombre fini d'éléments communs.

Nous nous occuperons ici seulement des familles $F$ d'ensembles infinis de nombres naturels.

THéonÈne 1. Il existe une famille dénombrable $F$ d'ensembles infinis de nombres naturels ayant deux d̀ deux au plus un élément commun et telle qu' il n'existe aucun ensemble qui ait avec tout ensemble de la famille $F$ un et un seul élément commun.

Démonstration. Soit $p_{n}$ le $n$-ième nombre premier. Soit

$$
E_{1}=\left\{2,2^{2}, 2^{3}, \ldots\right\}, \quad E_{2}=\left\{3,3^{2}, 3^{3}, \ldots\right\}
$$

On sait que tout nombre naturel $n$ peut être mis d'une seule manière sous la forme $n=2^{k_{n}-1}\left(2 l_{n}-1\right)$, où $k_{n}$ et $l_{n}$ sont des nombres naturels.

Posons, pour $n=1,2,3, \ldots$,

$$
E_{n+2}=\left\{2^{k_{n}}, 3^{l_{n}}, p_{n+2}, p_{n+2}^{2}, p_{n+2}^{3}, \ldots\right\} \text {. }
$$

Nous démontrerons que la famille $F=\left\{E_{1}, E_{2}, \ldots\right\}$ satisfait à notre théorème.

Les ensembles $E_{1}$ et $E_{2}$ sont disjoints. Or, il est évident que chacun des ensembles $E_{1}$ et $E_{2}$ a avec chaque ensemble $E_{n+2}(n=1,2, \ldots)$ un et un seul élément commun. Si l'on admet que les ensembles $E_{n+2}$ et $E_{m+2}$ 